\title{
Using instantaneous phase coherence for signal extraction from ambient noise data at a local to a global scale
}

\author{
M. Schimmel, ${ }^{1}$ E. Stutzmann ${ }^{2}$ and J. Gallart ${ }^{1}$ \\ ${ }^{1}$ Institute of Earth Sciences Jaume Almera - CSIC, Lluis Sole i Sabaris s/n, 08028 Barcelona, Spain. E-mail: schimmel@ictja.csic.es \\ ${ }^{2}$ Institut de Physique du Globe de Paris, UMR-CNRS 7154, PRES Sorbonne Paris Cite, 1 rue Jussieu, 75005 Paris, France
}

Accepted 2010 October 20. Received 2010 October 19; in original form 2010 April 16

\begin{abstract}
S UMMAR Y
Stacks of ambient noise cross-correlations are more and more routinely used to extract empirical Green's functions between station pairs. The success of the cross-correlations is due to waves which are recorded by both stations and that constructively sum at lag times which equal their propagation time between the station pair. Stacking cross-correlograms corresponding to different time spans improves the azimuthal noise coverage and further enhances the signals. Here we show how the instantaneous phase coherence can be used for a more efficient signal extraction from ambient noise cross-correlations. The instantaneous phase coherence is obtained by analytic signal processing and can be employed through the phase cross-correlation and/or through the time-frequency domain phase-weighted stack. The phase cross-correlation is more sensitive to waveform similarity but less sensitive to strong amplitude features than the conventional cross-correlation. The time-frequency domain phase-weighted stack cleans the ambient noise cross-correlograms by attenuating incoherent noise and permits an improved signal identification. We show that both approaches are powerful tools in the recovery of signals from ambient noise data and show examples where they improve the extraction of $P$ and Rayleigh waves by considering local and global scale applications.
\end{abstract}

Key words: Time series analysis; Interferometry; Body waves; Surface waves and free oscillations.

\section{INTRODUCTION}

Green's functions can be extracted from seismic noise crosscorrelations as theoretically shown with different approaches by Lobkis \& Weaver (2001), Derode et al. (2003), Wapenaar (2004), Snieder (2004), Roux et al. (2005a), Wapenaar et al. (2006), Snieder et al. (2007) among others. The Green's function recovery from noise fluctuations has now received much attention. A large amount of recent publications show the different applications in fields such as ultra sound (Weaver \& Lobkis 2001; Derode et al. 2003) helio seismology (Duvall et al. 1993; Rickett \& Claerbout 1999, 2000), fault and volcano monitoring (Sens-Schönfelder \& Wegler 2006; Wegler \& Sens-Schönfelder 2007; Brenguier et al. 2008), exploration seismology (Schuster et al. 2004; Bakulin \& Calvert 2006), crustal seismology (Campillo \& Paul 2003; Shapiro \& Campillo 2004; Shapiro et al. 2005; Sabra et al. 2005) and global seismology (Nishida et al. 2009). By now there exist many other noise studies which all have the main purpose to perform passive monitoring and/or structural imaging. Indeed, great promises are shown in this quickly developing field which opens up new applications.

Ideally the cross-correlations can only retrieve the Green's functions for systems with equipartitioned waves, that is, where energy is balanced such that its net flux becomes zero. In practice, however, this is not the case for most seismic ambient noise studies since the waves are not sufficiently scattered, since the noise sources are strongest along certain azimuths and since there are only finite number of independent sources. Averaging the cross-correlations over long time spans is commonly used to overcome these problems and to improve the emergence of the Green's function.

In a simplified manner, the ambient noise cross-correlations between two sensors can be pictured as a detection tool of waves which on their journey travel through both sensor positions. The crosscorrelation identifies these waves as a function of lag-time which is the traveltime from one sensor to the other. The presence of a significant number of waves which travel through the same path between the two stations and which can have different waveforms will lead to constructive contributions in the cross-correlation at given lag-time. The waveform details are lost by the cross-correlation and signals are build up which can be understood as due to waves which are generated by an impulsive source at one of the receiver locations. The positive or negative lag-time provides the information from which receiver these waves come from.

Based on this simplified picture one expects to detect these waves also with other signal detection tools which may even replace the commonly used cross-correlation and stacking techniques. Alternative signal detection approaches may retrieve complementary pieces of information and enable us to extract signals in a more efficient way. Efficient signal extraction is important for monitoring 
purposes since faster emerging signals can be detected using smaller time windows and can therefore improve the time resolution. It may also be possible to extract more signals from cross-correlograms due to an improved incoherent noise attenuation.

Due to this motivation we look for new processing techniques and show the performance of the phase cross-correlation (PCC) by Schimmel (1999) on ambient noise data. PCC is based on analytic signal theory and we show that since PCC is amplitude-unbiased, it does not require pre-processing steps such as the 1-bit normalization (e.g. Bensen et al. 2007) to remove the disturbing influence of energetic features such as earthquakes. The classical cross-correlation requires a pre-processing of the noise data which can deteriorate waveform coherence. The PCC of non-pre-processed noise data can therefore be more efficient than the classical cross-correlation as shown in our examples. Besides the correlations, the stacking of the cross-correlograms is further important to additionally improve the emergence of signals through their coherent appearance. Here we employ the time-frequency domain phase-weighted stack (tf-PWS) by Schimmel \& Gallart (2007). The tf-PWS strategy has also been used by Baig et al. (2009) to denoise ambient noise cross-correlograms for a more efficient Rayleigh- and Love-wave extraction.

In the following, we first recall the PCC (Schimmel 1999) and tf-PWS by Schimmel \& Gallart (2007) which then are applied to theoretical and real noise data. We show how to apply these tools and discuss benefits and limitations of our processing tools. Other advanced processing techniques may also be used in a similar fashion and for a robust ambient noise processing.

\section{METHODS}

The main ambient noise processing steps are (1) pre-processing, (2) cross-correlation and (3) cross-correlogram stacking. Here, we focus on the latter two and show how the instantaneous phase coherence obtained from the analytic signal can be incorporated in the ambient noise processing. In the following, we briefly summarize the main concept of the phase cross-correlation (PCC) and of the tf-PWS which then are used to correlate and stack ambient noise data. The correlation results are compared with the classical cross-correlation which is geometrically normalized (CCGN)

$c_{\mathrm{ccgn}}(t)=\frac{\sum_{\tau=\tau_{0}}^{\tau_{0}+T} u_{1}(t+\tau) u_{2}(\tau)}{\sqrt{\sum_{\tau=\tau_{0}}^{\tau_{0}+T} u_{1}(t+\tau)^{2} \sum_{\tau=\tau_{0}}^{\tau_{0}+T} u_{2}(\tau)^{2}}}$,
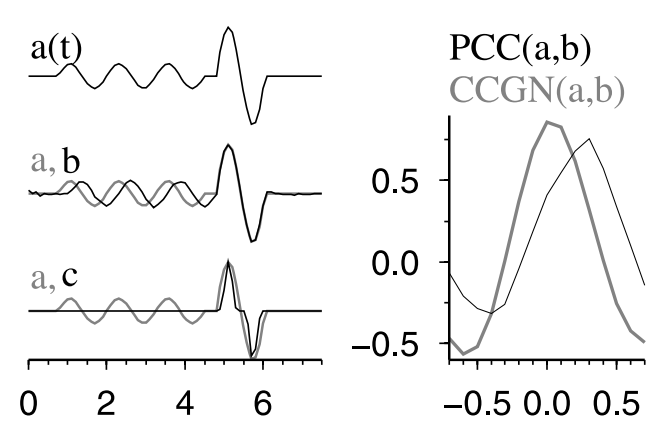

a) Time $[\mathrm{s}]$

b)

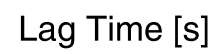

where $u_{1}$ and $u_{2}$ are the two time-series and where $t$ and $T$ are the time lag and correlation window length, respectively. $\tau_{0}$ is the start time $t-T / 2$ of the correlation window. The time lag $t$ is applied to $u_{1}$. The denominator is the geometric mean of the energy within time window $T$.

\subsection{The PCC}

The PCC is based on analytic signal theory. The analytic signal $s(t)$ of a real time-series $u(t)$ is uniquely defined as $s(t)=u(t)+$ $i H[u(t)]$, where $H[u(t)]$ is the Hilbert Transform of the time-series $u(t)$. It is a transformation from the real to the complex number domain. Considering the exponential form $s(t)=a(t) \exp (i \Phi(t))$ one obtains the envelope $a(t)$ and the instantaneous phase $\Phi(t)$. It is this phase that should be the same for coherent signals at each given time. The waveform information of the neighbouring samples is contained implicitly through the Hilbert Transform which one can express as a heavily localized moving window operation. PCC was introduced in Schimmel (1999) and is defined as

$c_{\text {pcc }}(t)=\frac{1}{2 T} \sum_{\tau=\tau_{0}}^{\tau_{0}+T}\left\{\left|\mathrm{e}^{\mathrm{i} \Phi(t+\tau)}+\mathrm{e}^{\mathrm{i} \Psi(\tau)}\right|^{\nu}-\left|\mathrm{e}^{\mathrm{i} \Phi(t+\tau)}-\mathrm{e}^{\mathrm{i} \Psi(\tau)}\right|^{\nu}\right\}$.

$c_{\text {pcc }}$ is a coherence functional which measures the similarity of two time-series $u_{1}$ and $u_{2}$ as function of lag time $t$ in analogy to the classical cross-correlation (eq. 1). $\Phi(\tau)$ and $\Psi(\tau)$ are the instantaneous phases of $u_{1}$ and $u_{2}$ which make $c_{\text {pcc }}(t)$ an amplitude unbiased correlation. PCC is normalized to $\left|c_{\mathrm{pcc}}\right| \leq 1$ with $c_{\mathrm{pcc}}=$ 1 indicating perfect correlation and $c_{\mathrm{pcc}}=-1$ anticorrelation. The sensitivity of $c_{\text {pcc }}(t)$ can be increased by using power $v>1$ (eq. 2 ). This may lead to an improved signal-to-noise ratio (SNR) while absolute correlation values may decrease. We use $v=1$ throughout this work.

$c_{\mathrm{pcc}}$ is based on the number of phase coherent samples rather than the sum of products as it is the case for the conventional cross-correlation. Fig. 1 shows how the different concepts may influence the correlation results. The example uses time-series $a(t)$, $b(t)$ and $c(t)$ which are shown as black lines in Fig. 1(a). $a(t)$ and $b(t)$ consist of two wave trains each. The only difference between $a(t)$ and $b(t)$ is that the first wave train is delayed by about $0.3 \mathrm{~s}$ on $b(t)$. PCC and CCGN of traces $a(t)$ and $b(t)$ are shown as black and grey line in Fig. 1(b). The two methods have maximum correlation at different lag times. CCGN has its maximum at zero lag advocating best waveform similarity through aligning the large amplitude

Figure 1. (a) Black lines show the time-series $a(t), b(t), c(t)$ used in subsequent illustration. $b(t)$ and $c(t)$ are plotted together with $a(t)$ (grey line) to simplify the visual inspection of waveform coherence. (b) The black (PCC) and grey line (CCGN) are the cross-correlations of $a(t)$ with $b(t)$. The lag time is applied to $a(t)$. The different maxima at different lag point to different waveform similarity. (c) Shown are the correlations of $a(t)$ with $b(t)$ (grey lines) and $a(t)$ with $c(t)$ (black lines). CCGN is less sensitive than PCC to the waveform changes. 
signal at about $5 \mathrm{~s}$ (Fig. 1a). PCC finds best waveform alignment at lag $0.3 \mathrm{~s}$ that is by aligning the smaller amplitude wave train of $3 \mathrm{~s}$ duration. PCC is amplitude insensitive and determines the maximum correlation by the maximum number of coherent samples. That is, the $0.3 \mathrm{~s}$ lag time is favoured since it aligns the longer wave train. The CCGN does not favour the alignment of this wave train since the sum of products is largest by aligning the largest amplitude signals, thus yielding maximum correlation at zero lag. As consequence, CCGN is the better choice when looking for the most energetic signals. Fig. 1(c) illustrates the cross-correlograms for traces $a(t), b(t)$ (grey line) and $a(t), c(t)$ (black line). $c(t)$ contains only the large amplitude signal of trace $a(t)$ with changed waveform. Striking is the similarity of the CCGN correlograms which shows that the small amplitude portions have little influence in the cross-correlation. Conversely, the PCCs have different lag time and amplitudes. The lag times for maximum correlation are $0.3 \mathrm{~s}$ and $0 \mathrm{~s}$ for traces $a(t), b(t)$ and $a(t), c(t)$ and show the preferred alignment of the small amplitude wave train and of the large amplitude signal, respectively. The amplitude of maximum correlation for traces $a(t)$, $c(t)$ and $a(t), b(t)$ are 0.353 and 0.755 , respectively. As expected, the amplitudes of the cross-correlation values are smaller for $a(t)$, $c(t)$ since their waveforms are less similar. PCC is more sensitive to waveform changes which depending on data and application in mind can be an advantage or disadvantage. Anyhow, PCC is an independent coherence measure based on a different definition for waveform similarity and which can be more useful in discriminating closely similar waveforms.

\subsection{The time-frequency domain PWS}

The tf-PWS is an extension of the PWS presented by Schimmel \& Paulssen (1997). The PWS is a non-linear stack where each sample of a linear stack is weighted by an amplitude-unbiased coherence measure, the phase stack

$c_{\mathrm{ps}}(t)=\left|\frac{1}{N} \sum_{j=1}^{N} \mathrm{e}^{\mathrm{i} \Phi_{j}(t)}\right|^{\nu}$.

$c_{\mathrm{ps}}(t)$ is based on the similarity of the instantaneous phases $\Phi_{j}(t)$ of the complex signals of the $N$ traces $u_{j}(t)$ at time $t$. $v$ is a parameter to tune the transition between coherent and less coherent signal summation. In analogy, Schimmel \& Gallart (2007) present the tfPWS for array processing and adaptive filtering. The backbone of their tool is the time-frequency phase stack which is based on the time-frequency decomposition of each trace which they obtained through the $S$-transform (Stockwell et al. 1996)

$S(\tau, f)=\int_{-\infty}^{\infty} u(t) w(\tau-t, f) \mathrm{e}^{-\mathrm{i} 2 \pi f t} \mathrm{~d} t$

with a Gaussian window function $w(\tau-t, f)$ centred at time $\tau$ and width proportional to $|1 / f|$.

$w(\tau-t, f)=\frac{|f|}{k \sqrt{2 \pi}} \mathrm{e}^{\frac{-f^{2}(\tau-t)^{2}}{2 k^{2}}}, \quad k>0$.

The $S$-transform is a windowed Fourier Transform where the windows (amplitude and width) are frequency $(f)$ dependent. The windows and thus the resolution can be adjusted using variable $k$. We use $k=2$ in the following analysis. The $S$-transform is related to the wavelet transform through a matrix multiplication (Ventosa et al. 2008). Any other time-frequency decomposition method which results into an analytic representation of the time-series can be used in full analogy. The advantage of the $S$-transform is that it is based on the well-known Fourier theory. In Schimmel \& Gallart (2007) it is shown under which conditions the $S$-transform decomposition is analytic. In their eq. (13) and following two paragraphs, $k$ should stand in the numerator rather than the denominator. This writing mistake does not further change the physics or conclusions. It is shown that $S(\tau, f) \mathrm{e}^{\mathrm{i} 2 \pi f \tau}$ is analytic for any real signal at a fixed frequency $f$. Consequently, and in analogy to eq. (3) the time-frequency phase stack can be written as

$c_{\mathrm{ps}}(\tau, f)=\left|\frac{1}{N} \sum_{j=1}^{N} \frac{S_{j}(\tau, f) \mathrm{e}^{\mathrm{i} 2 \pi f \tau}}{\left|S_{j}(\tau, f)\right|}\right|^{\nu}$,

where $S_{j}(\tau, f)$ is the $S$-transform (eq. 4 ) of the $j$-th time-series (here cross-correlograms). That is to determine $c_{\mathrm{ps}}(\tau, f)$ each individual trace must be transformed into the time-frequency domain. The tfPWS $S_{\text {pws }}(\tau, f)$ is obtained by a time-frequency multiplication of the phase stack $c_{\mathrm{ps}}(\tau, f)$ with $S_{\mathrm{ls}}(\tau, f)$ the $S$-transform of the linear stack of all $N$ time-series (cross-correlograms).

$S_{\mathrm{pws}}(\tau, f)=c_{\mathrm{ps}}(\tau, f) S_{\mathrm{ls}}(\tau, f)$.

The phase coherence $c_{\mathrm{ps}}(\tau, f)$ is thus used to downweight the incoherent portions of the linear stack in the time-frequency domain. The inverse $S$-transform (Schimmel \& Gallart 2005, 2007; Simon et al. 2007) is then applied to transform $S_{\mathrm{pws}}(\tau, f)$ to the time domain $s_{\text {pws }}(t)$. In Schimmel \& Gallart (2007) it is shown how the phase stack can be used as local weight (in space, time, slowness and frequency) in record sections or array data. Baig et al. (2009) employ this tf-PWS based on a non-redundant and efficient version of the $S$-transform.

\section{APPLICATION TO NOISE DATA}

\subsection{Synthetic data: comparison of cross-correlations}

In the following we use synthetic data to give an overview on the performance and limitations of the different correlation approaches. Using synthetic data has the advantage that one can determine the efficiency from controlled experiments with known signals and noise before applying the methods to real data. Our synthetic data are kept as simple as possible without intention to mimic real ambient noise. Real ambient noise are very complicated due to the different noise generation mechanisms and complex wave propagation in heterogeneous media. Here we are only interested in studying the detection of waves which travel in one direction and which are contaminated by incoherent background noise and a single strong earthquake.

Earth ambient noise is mainly build up with dispersive surface waves due to the dominant ubication of noise sources on the Earth surface. Our test data consist of many surface wave trains, random noise and optionally one earthquake. The purpose is to show how the surface wave signal can be extracted from the data. We assume that the dispersive surface waves are propagating without loss of energy along the path between two stations. We add background noise to perturb randomly the waveforms. The background noise is not assumed to be white. The SNR and number of dispersive surface waves can be controlled in our test data which are explained with more details in the following.

\subsubsection{Generating test data}

A large portion of the ambient noise data consist of dispersive surface waves and we therefore chose to generate synthetic test data with dispersive signals to mimic surface wave propagation. 

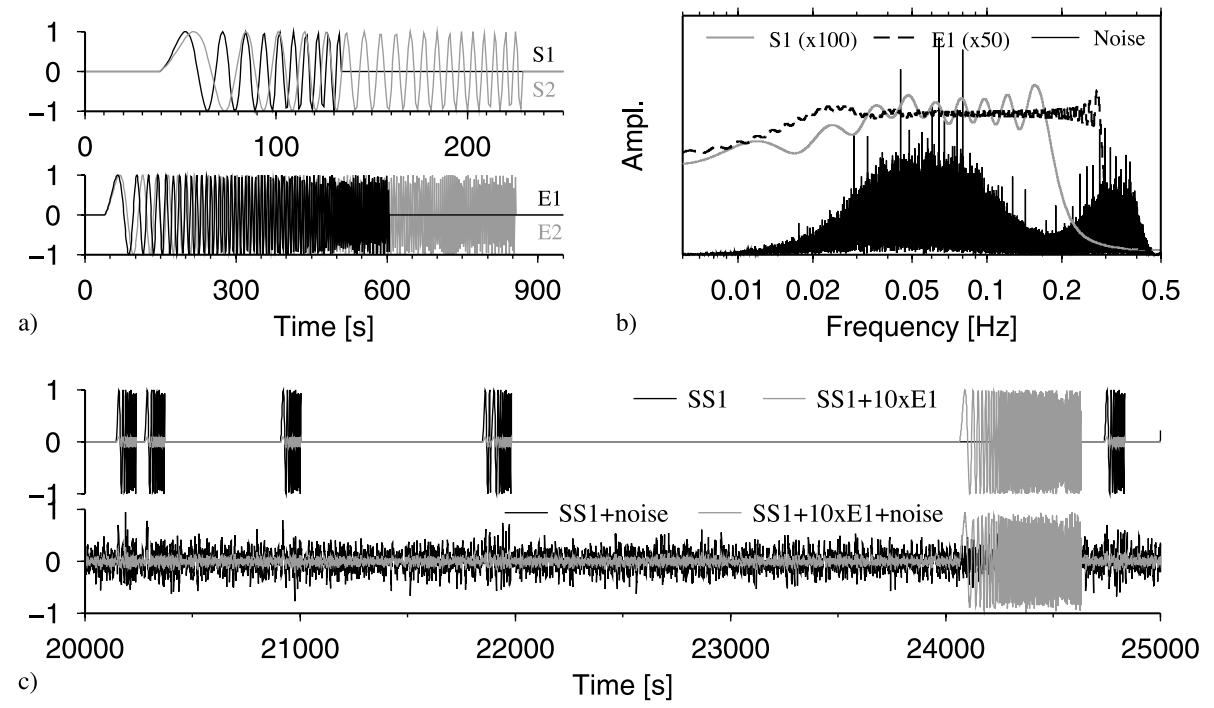

Figure 2. (a) $S 1, S 2, E 1, E 2$ are the waveforms used to construct our test series. The Fourier amplitude spectra of $S 1$ and $E 1$ are shown in (b). The amplitude scale is linear with relative amplitudes marked in the figure. (c) Grey and black lines show a $5000 \mathrm{~s}$ zoom of the synthetic traces used in subsequent correlation tests. The total length of the time-series is $1 \mathrm{~d}$. The top panel and bottom panel show the noise-free and noise-contaminated traces. The noise amplitude spectrum is shown in (b).

Test data have been generated using two sinusoidal linear chirp functions $S_{1}(t)$ and $S_{2}(t)$

$S_{i}(t)=\left\{\begin{array}{ll}\sin (2 \pi \tilde{f} t) & \text { if } t>0 \text { and } \tilde{f}=f_{0}+r_{i} t<0.1 \mathrm{~Hz} \\ 0 & \text { otherwise, }\end{array}\right.$,

where $i$ is 1 or 2 , and where $\tilde{f}$ is obtained with $f_{0}=0.005 \mathrm{~Hz}, r_{1}=$ $0.001 \mathrm{~s}^{-2}$ and $r_{2}=0.0005 \mathrm{~s}^{-2}$. The Fourier amplitude spectrum of signal $S_{1}$ is shown in Fig. 2(b) (grey line). As can be seen from the equations and Fig. 2(a), the instantaneous frequency $\tilde{f}$ of the signals increase linearly with time and the two chirp signals have a different frequency dispersion so that the different frequency components arrive at different time. This mimics dispersive surface waves which have been recorded at two different stations. Due to the dispersion, the cross-correlogram of these chirp functions should show different lag times as function of frequency which correspond to the wave propagation time between both stations.

The first test trace $S S_{1}(t)$ (Fig. 2c) is constructed by distributing the chirp $S_{1}(t)$ randomly hundred times over a day. The second test trace $S S_{2}(t)$ is obtained by shifting the randomly distributed chirps by $100 \mathrm{~s}$ and by replacing chirp $S_{1}(t)$ by chirp $S_{2}(t)$, that is,

$S S_{i}(t)=\sum_{k=1}^{100} S_{i}\left(t-t_{k}-t_{i}\right)$,

with $t_{k} \in[0 \mathrm{~s}, 86400 \mathrm{~s}], t_{1}=0$ and $t_{2}=100 \mathrm{~s}$.

To simulate a large event, such as an earthquake, we construct in addition two chirps $E_{1}(t)$ and $E_{2}(t)$ using eq. (8) and parameters $f_{0}=0.003 \mathrm{~Hz}, r_{1}=0.00026 \mathrm{~s}^{-2}, r_{2}=0.00018 \mathrm{~s}^{-2}$ and $\tilde{f}<0.15 \mathrm{~Hz}$. We multiply $E_{1}(t)$ and $E_{2}(t)$ by a factor of 10 and use these chirps to mimic a large event that contaminates at random time $t_{e}$ and $t_{e}+40 \mathrm{~s}$ the test data $S S_{1}(t)$ and $S S_{2}(t)$, respectively. The chirp functions $E_{1}(t)$ and $E_{2}(t)$ have different dispersions with the lowest frequencies arriving first with $40 \mathrm{~s}$ lag time. The normalized traces $S S_{1}(t)$ and $S S_{1}(t)+10 E_{1}\left(t-t_{e}\right)$ are shown in Fig. 2(c) with and without random noise contamination. The background noise is not white as it is for real noise spectra. Its shape is chosen arbitrarily with amplitude spectrum shown in Fig. 2(b). The background noise perturb randomly the signal waveforms. The Nyquist frequency of our test data is $0.5 \mathrm{~Hz}$.

\subsubsection{Pre-processing}

Our pre-processing is kept simple and leans on the commonly used pre-processing (e.g. Bensen et al. 2007) for the computation of ambient noise cross-correlations. It consists in performing a 1-bit normalization and a spectral whitening of the traces. The 1-bit normalization $\left(\frac{u(t)}{|u(t)|}\right)$ removes waveform details and amplitude differences in the time domain by dividing at each time the recorded amplitude by its absolute amplitude. Through this operation the cross-correlation is made amplitude insensitive. It means that large amplitude signals such as strong earthquakes should not govern the correlation through their large amplitudes.

We perform the spectral whitening after application of the 1-bit normalization. The time-series are transformed to the frequency domain to normalize their amplitude spectra to 1 . The phase spectra are not changed. The amplitude normalization in the frequency domain is useful to remove the influence of signals which manifest in amplitude spectra such as frequency-localized noise sources. The 26 s resonance (Holcomb 1998) in the Gulf of Guinea as localized by Shapiro et al. (2006) is such signal which can be removed by the whitening (Bensen et al. 2007). The normalized spectra are then transformed back to the time domain. We apply no bandpass filter to our synthetic data since there are signals almost throughout the entire frequency range. However, we apply a bandpass filter to the real data to reduce the frequency interval where one may expect to extract signals from coherent noise. The frequency range to select depends also on the interstation distance. Cross-correlations performed at dominate frequencies with corresponding wave lengths larger than the interstation distance may lead to a perfect correlation between the two stations and converge to the autocorrelation (Chavez-Garcia \& Rodriguez 2007). For far-field Green's functions the interstation distance should be several times larger than the wavelength. 


\subsubsection{Cross-correlations}

The cross-correlations between synthetic traces $S S_{1}(t)$ and $S S_{2}(t)$ with or without noise contamination, with or without pre-processing are shown in Fig. 3. The four upper and lower panels show the results for CCGN and PCC, respectively. The cross-correlograms of Fig. 3(a) are unfiltered while those of Fig. 3(b) have been bandpassed from $0.05 \mathrm{~Hz}$ to $0.2 \mathrm{~Hz}$. The black and grey lines are used to distinguish the correlations for the noise-free and noisy data, respectively. Further, the label $E+$ marks that the chirp functions $E_{1}(t)$ and $E_{2}(t)$ were used to contaminate both test traces to simulate the occurrence of a large event. Label $E-$ means that no event contaminates the test data. Similarly, $W+$ and $W-$ are used to indicate whether the data have been pre-processed following the steps described in the previous subsection.

The first line of Fig. 3 shows with black line the CCGN crosscorrelogram for the noise-free traces without pre-processing $(W-)$ and without the large event contamination $(E-)$. Since this is the cross-correlation of noise-free data the pre-processing is not required and the correlation result is what one can ideally expect to achieve for the following cross-correlograms of the differently manipulated data. The dispersive signal shape is due to the different dispersions of the chirps which result in increasing lag times for increasing frequencies. The lowest frequency component has a correct lag time of $100 \mathrm{~s}$. In the second line we show the cross-correlations for the same, but pre-processed $(W+)$ traces. The results seem to be contaminated with high-frequency noise. The spectral normalization (whitening) increased the higher frequency amplitudes due to the absence of high-frequency signals. The third line contains the results for $E+, W-$. Here we see the effect of the strong amplitude chirp which starts to correlate at lag $40 \mathrm{~s}$ for the lowest frequency components. The $40 \mathrm{~s}$ lag corresponds to the time of the shifted strong amplitude chirp $E_{2}(t)$ with respect to $E_{1}(t)$. The correlation at $t>40 \mathrm{~s}$ is due to the different dispersion of the chirps $E_{1}(t)$ and $E_{2}(t)$. The correlation of the 10 times smaller amplitude chirps interferes with the strong event correlation at lags $100 \leq t<200 \mathrm{~s}$. The fourth line shows the correlation of the same data but with $W+$. It can be seen that the traces CCGN, $E-, W+$ and CCGN, $E+, W+$ are quite similar thus insensitive to the strong event $E+$. That is the 1-bit normalization and whitening fulfill their purpose of neutralizing the occurrence of strong events in the time and frequency domain. The pre-processing is therefore important to remove these signatures from the input data. The comparison of the filtered cross-correlograms CCGN, $E-, W-$ and CCGN, $E+, W+$ however show that the removal of the strong event through $W+$ may introduce waveform perturbations.

The following four panels (Fig. 3) show the PCC results for the data used in the first four lines. The noise-free and unfiltered correlations PCC, $E-, W-$ and PCC, $E+, W-$ have a high correlation at all lags as can be seen from Fig. 3(a) (5th and 7th black trace). This is due to the zero amplitude portions of the noise-free data which also are phase coherent. The filtered correlations PCC, $E-, W-$ and PCC, $E+, W-$ of the noise-free data are also affected by the zero amplitude portions. It can be seen from Fig. 3 that the inclusion of some noise (grey lines) remove this high average correlation. In practice, real data have no zero amplitude stretches and will therefore not show the overall high correlation. Striking is the similarity of the correlation waveforms PCC, $E-, W-$ and PCC, $E+, W-$. It shows that $\mathrm{PCC}$ is not sensitive to the high amplitude event $E+$. This is due to the strategy of PCC which has been designed as an amplitude unbiased approach based on the instantaneous phase coherence. The number of phase-coherent samples for $E_{i}(t)$ is in the order of 100 times less than for the small amplitude chirp $S_{i}(t)$. The comparison of PCC, $E-, W+$, PCC, $E+, W+$ with PCC, $E-$,
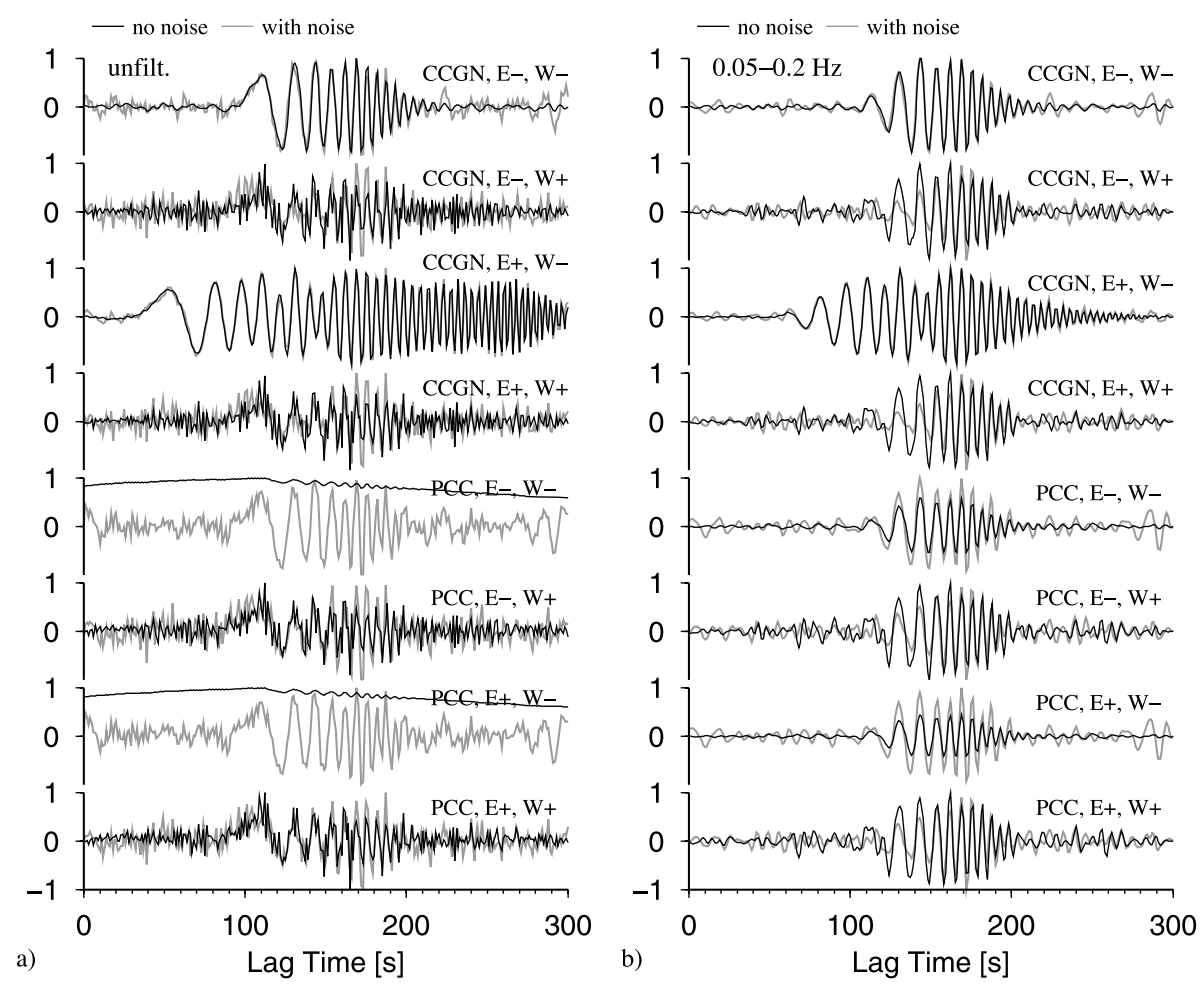

Figure 3. (a) Black and grey lines show the correlation results for noise-free and noise-contaminated traces (Fig. 2c, either upper or lower panel), respectively. $E+$ and $E$ - indicate the inclusion or absence of a large amplitude event and $W+$ and $W$-indicate whether the pre-processing included 1-bit normalization and spectral whitening or not. (b) Same as (a) but the data have been bandpassed between 0.05 and $0.2 \mathrm{~Hz}$. 
$W-$ (or CCGN, $E-, W-$ ) show that whitening $(W+)$ can degrade the phase coherence through waveform perturbations. This is also seen from the filtered cross-correlograms of Fig. 3(b). The bandpass filter removes signals due to weighting outside the dominant signal frequency band which increases the SNR of the cross-correlograms. In real data the frequency band of coherent signals in the ambient noise is unknown a priori and often difficult to access.

From this analysis, we conclude that the pre-processing can deteriorate the correlations, but that the pre-processing is required to obtain satisfactory results with CCGN whenever one can expect large amplitude events in the data. PCC has the advantage that it does not need the pre-processing but since it is based on phase coherence it may be more sensitive to waveform variations of the signals one is looking for.

\subsubsection{Robustness of cross-correlation results}

We now re-do the cross-correlations for different background noise realizations to investigate the robustness of the results. As background noise we name the omnipresent noise in time with amplitude spectrum shown in Fig. 2(b). The degree of noise contamination is quantified through the SNR which we define using the rms amplitude of the noise-free signal considering the non-zero amplitude portions of $S S_{1}(t)$ and the rms amplitude of the background random noise.

$\mathrm{SNR}=20 \log _{10}\left(\frac{\mathrm{rms}_{\text {signal }}}{\mathrm{rms}_{\text {noise }}}\right)$.

The logarithmic decibel scale (eq. 10) is employed for visual purpose. The cross-correlations are computed for different random noise realizations and different SNR. We measure the similarity between the various PCC and CCGN waveforms by the amplitude of the zero-lag cross-correlation of each waveform with the noisefree CCGN, $E-, W$ - for times between $100 \mathrm{~s}$ and $175 \mathrm{~s}$. Each crosscorrelation result is represented as a dot as function of its similarity with the noise-free CCGN, $E-, W-$ and SNR in Fig. 4. The colours identify the correlation type (CCGN or PCC) and the pre-processing $(W-$ or $W+$ ). The estimated SNR of the test traces from Fig. 2 is $0.485 \mathrm{~dB}$ corresponding to an rms amplitude ratio of approximately 1.057. The difference between Figs 4(a) and (b) is that the data from Fig. 4(a) were not contaminated by a large amplitude event $(E-)$

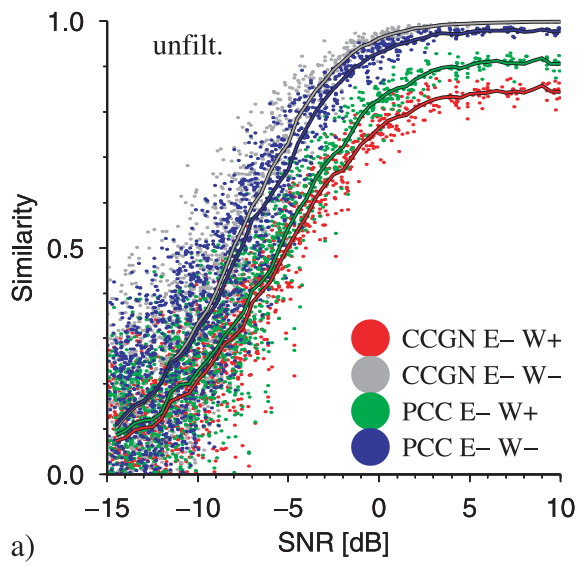

while the data from Fig. 4(b) were contaminated by a large event $(E+)$.

It can be seen from Fig. 4(a) that the CCGN without any data preprocessing (CCGN, $E-, W-$ ) provides the best results followed by the PCC (PCC, $E-, W-$ ). The PCC suffers more from variations in the waveform details through the noise contamination and therefore does not exactly reach 1 , or in other words similarity with the noisefree template CCGN, $E-, W-$ is not reached. The high waveform sensitivity also explains the larger similarity variations for PCC at positive SNR with respect to CCGN. The green and red dots correspond to the PCC and CCGN measure for the pre-processed data $(W+)$. PCC performs on average slightly better than the CCGN. The 1-bit normalization makes it more difficult to detect the chirps through the sliding inner products of the correlation.

Fig. 4(b) shows the results for the same data but contaminated additionally by a large amplitude event $(E+)$. Striking, but expected, is the poor performance of the CCGN, $E+, W-$. The correlation is strongly biased by $E+$ and pre-processing $W+$ is required. As expected from Fig. 3, the other cross-correlations are not significantly changed by the pre-processing. Now, PCC provides clearly the best results. Performing the same analysis on the $0.05-0.2 \mathrm{~Hz}$ bandpassed data is not changing the results from Fig. 4.

The cross-correlation results depend also on the number of signals included in the record. The following figure (Fig. 5) shows the zero-lag CCGN similarity of the cross-correlograms as function of number of signals $\left(S_{i}\right)$ spread randomly over $1 \mathrm{~d}$. The SNR of the data has been fixed for this figure with an average and standard deviation of $0.41 \pm 0.31 \mathrm{~dB}$. 100 signals spread over the day cover roughly 10 per cent of the day time. Figs 5(a) and (b) show the results for $E-$ and $E+$, respectively. It can be seen from Fig. 5 that there exists a consistent pattern with respect to Fig. 4. CCGN, $E-, W-$ provide clearly the best result for no strong signal contamination (Fig. 5a). However, if there exists a strong amplitude event $(E+)$ then PCC, $E+, W-$ becomes the better approach since it is less sensitive to $E+$ than CCGN. This outcome seems to be independent of the number of signals considered. The results of Fig. 5(b) depend also on the length and amplitude of the strong amplitude event $E_{i}(t)$. The stronger this signal is, the less it is contaminated by the noise and the more its 1-bit pattern remains present after the pre-processing.

These tests show that PCC is a powerful method that can, under certain conditions, retrieve the expected template which was

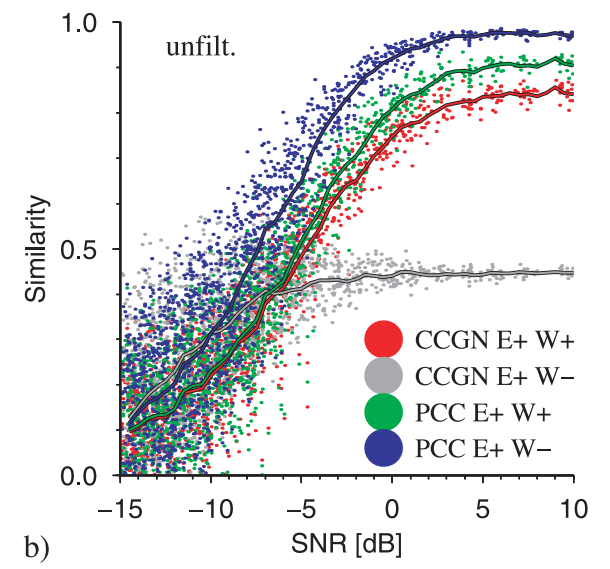

Figure 4. Cross-correlation results for the differently processed traces (Fig. 3a) are separated by the different colours. Each dot represents a result as function of data SNR (eq. 10) and its similarity with the noise-free data cross-correlogram (CCGN, $E-, W-$ ). The similarity is the zero-lag CCGN for $t \in[100 \mathrm{~s}, 175 \mathrm{~s}]$. Solid lines mark the local mean and a total of $4 \times 1940$ correlations were computed per figure. (a) and (b) show the results without $(E-)$ and with $(E+)$ strong event contamination, respectively. The SNR of our test data Fig. 3(a) is $0.485 \mathrm{~dB}$. 

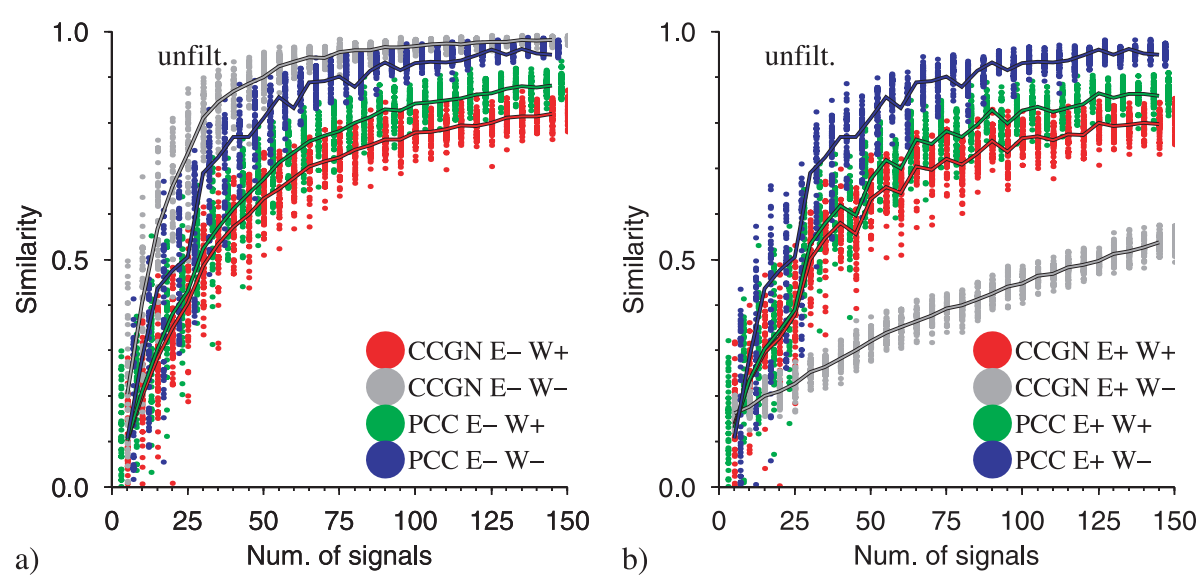

Figure 5. Cross-correlation results for the differently processed traces (Fig. 3a) which are separated by different colours. Each dot represents a result as function of number of signals in the input data and its similarity with the noise-free data cross-correlogram (CCGN, $E-, W-$ ). 100 signals cover about 10 per cent of the day. SNR is $0.41 \pm 0.31 \mathrm{~dB}$. Solid lines mark the mean and a total of $4 \times 1486$ correlations were computed per figure. The similarity is the zero-lag CCGN for $t \in[100 \mathrm{~s}, 175 \mathrm{~s}]$. The green and blue symbols were shifted horizontally by +2 and -2 to improve the inspection of similarity variability. (a) and (b) show the results without $(E-)$ and with $(E+)$ strong event contamination, respectively.

obtained from the noise-free cross-correlograms (CCGN, E-, $W-$ ). PCC was the better approach in retrieving this template when the data were contaminated by one large amplitude event. In this case CCGN needs the pre-processing to remove the amplitude bias but which may degrade signal waveforms in the noise. It is observed that on average PCC (with and without pre-processing) performs better than CCGN (Fig. 5b).

\subsubsection{Group velocities}

Finally, we analyse the cross-correlations in the time-frequency domain. Ambient noise cross-correlations are mostly used to extract the dispersion of surface waves through phase and group velocity measurements. Here we assume for simplicity that our synthetic cross-correlations are for two stations which are separated by $300 \mathrm{~km}$ and measure their group velocities. The group velocities are determined from the maxima of the time-frequency amplitude spectra. The $S$-transform (Stockwell et al. 1996) is used to obtain the time-frequency representation of the cross-correlograms. We plot group velocities for all maxima which are larger than 30 per cent of the extremum in each time-frequency representation. The cross-correlograms used, CCGN and PCC, are shown in Figs 6(a) and (b), respectively. These traces are the same as those shown in Fig. 2(a). Figs 6(c) and (d) show their corresponding group velocities. The legend in Fig. 6(d) is for the entire figure and the colours correspond to traces in Figs 6(a) and (b). The two different dispersions shown in Fig. 6(c) are due to the randomly distributed chirps $S_{i}(t)$ and the strong event $E_{i}(t)$. Their highest corner frequencies of about 0.18 and $0.3 \mathrm{~Hz}$ correspond to the highest resolved chirp corner frequencies shown in Fig. 2(b). This figure shows that $E+$ dominates the cross-correlation over all frequencies for the CCGN approach without applied pre-processing (CCGN, $E+, W-$ ). The higher frequency noise (isolated maxima at frequencies larger than $0.2 \mathrm{~Hz}$ ) belong to the cross-correlations of the pre-processed $(W+)$ data. Their SNR in the time-frequency domain is decreased due to the whitening and these maxima enter through the chosen 30 per cent threshold.

Fig. 6(d) shows the dispersion curves for PCC. It is striking that all the measured group velocities resemble each other which confirms that the results are not influenced by the strong event $E+$.
Further, there appear also other maxima at the higher frequencies which are again explained by the decrease of the SNR caused by $W+$ and the chosen 30 per cent threshold.

\subsection{Real data: high-frequent regional-scale cross-correlations}

We now employ the data from a temporary broad-band array which has been deployed during $1 \mathrm{yr}$ in the province of Almeria (South Spain) to investigate the deep Alboran area with advanced array processing. A map of the array is shown in Fig. 7. The array has an aperture of about $35 \mathrm{~km}$ and consists of 10 stations which are irregularly placed with interstation distances ranging from $2.5 \mathrm{~km}$ to $35 \mathrm{~km}$. In the following we show the performance of the described tools in the detection of high-frequency surface waves and body waves.

We use continuous vertical component recordings which we cut into $24 \mathrm{~h}$ traces to later perform cross-correlations on daily records for all possible station pairs. The data were bandpass filtered between 0.3 and $3 \mathrm{~Hz}$. No additional pre-processing was applied for PCC in harmony to our synthetic data results. For CCGN the data were pre-processed using the time-domain and frequency-domain whitening as described in the previous section. The mentioned bandpass filter was applied after the spectral whitening. The daily cross-correlograms, either CCGN or PCC, were stacked linearly and non-linearly using tf-PWS (eq. 7) for each station pair. The final cross-correlograms are based on the data of a minimum of $20 \mathrm{~d}$ to a maximum of $110 \mathrm{~d}$, depending on data availability. The cross-correlations are performed such that positive lag is applied to the station to the South. Most of the sources are expected to be in the Mediterranean Sea and will cause larger amplitude signals at positive lag.

In Fig. 8, we show the cross-correlograms as function of interstation distance and lag time. The linear stack of the daily CCGN and PCC traces are shown in Figs 8(a) and (b). The Rayleigh-wave arrival is seen as aligned signals at negative and positive lag time. The waves are better visible at positive lag due to the dominance of sources in the Mediterranean Sea. The stations were located in a complicated mountain area (Sierra Nevada mountain range) which 
a)
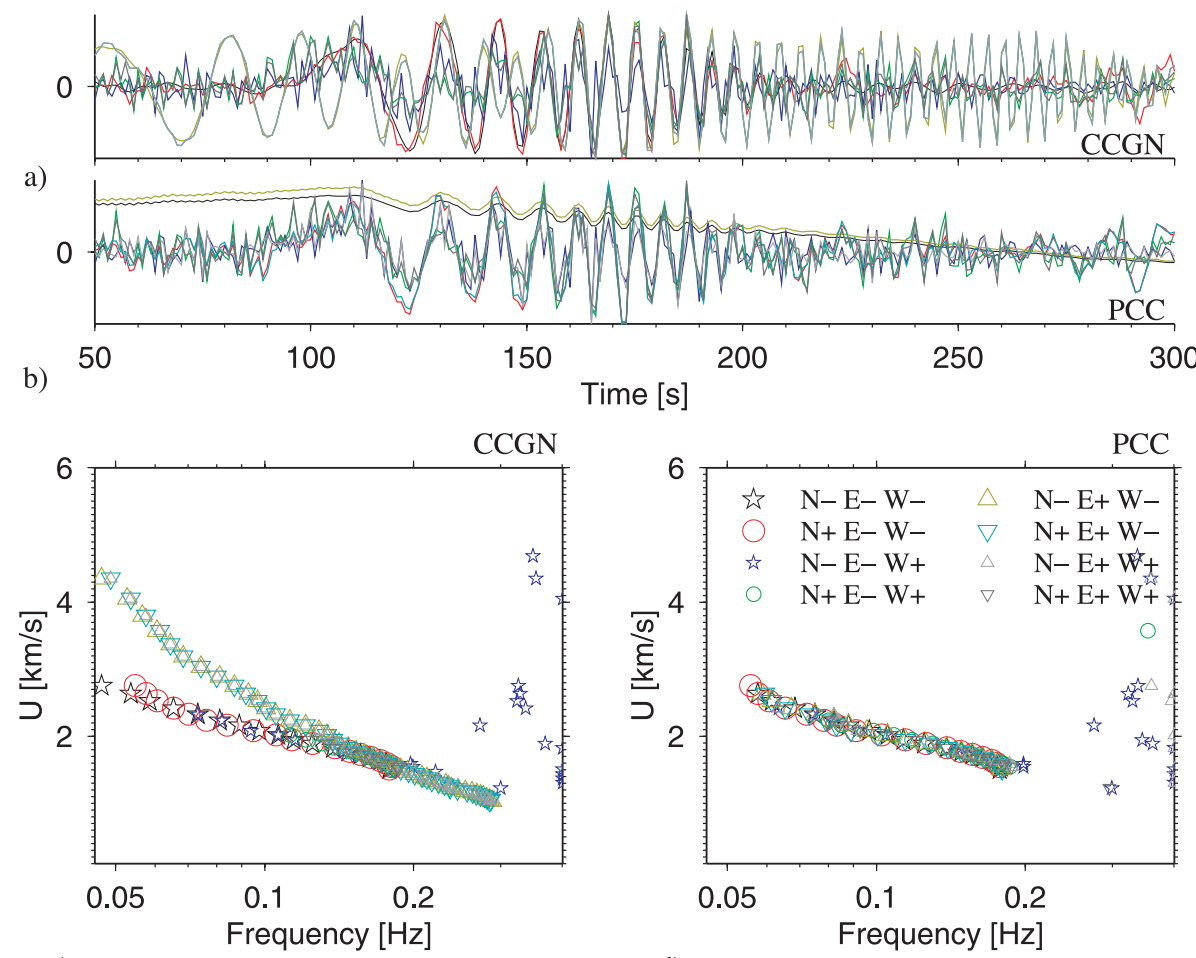

c)

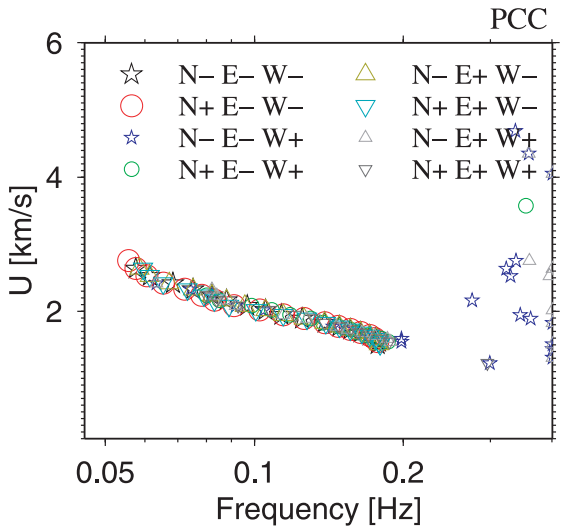

d)

Figure 6. (a,b) The cross-correlograms from Fig. 3(a) using CCGN and PCC, respectively, are plotted on top of each other. The colours correspond to the labels of (d). (c) and (d) show the measured group velocities for the CCGN and PCC in (a) and (b). N+ and $N-$ stand for noise-contaminated and noise-free data. $W$ and $E$ are used as in Fig. 3.

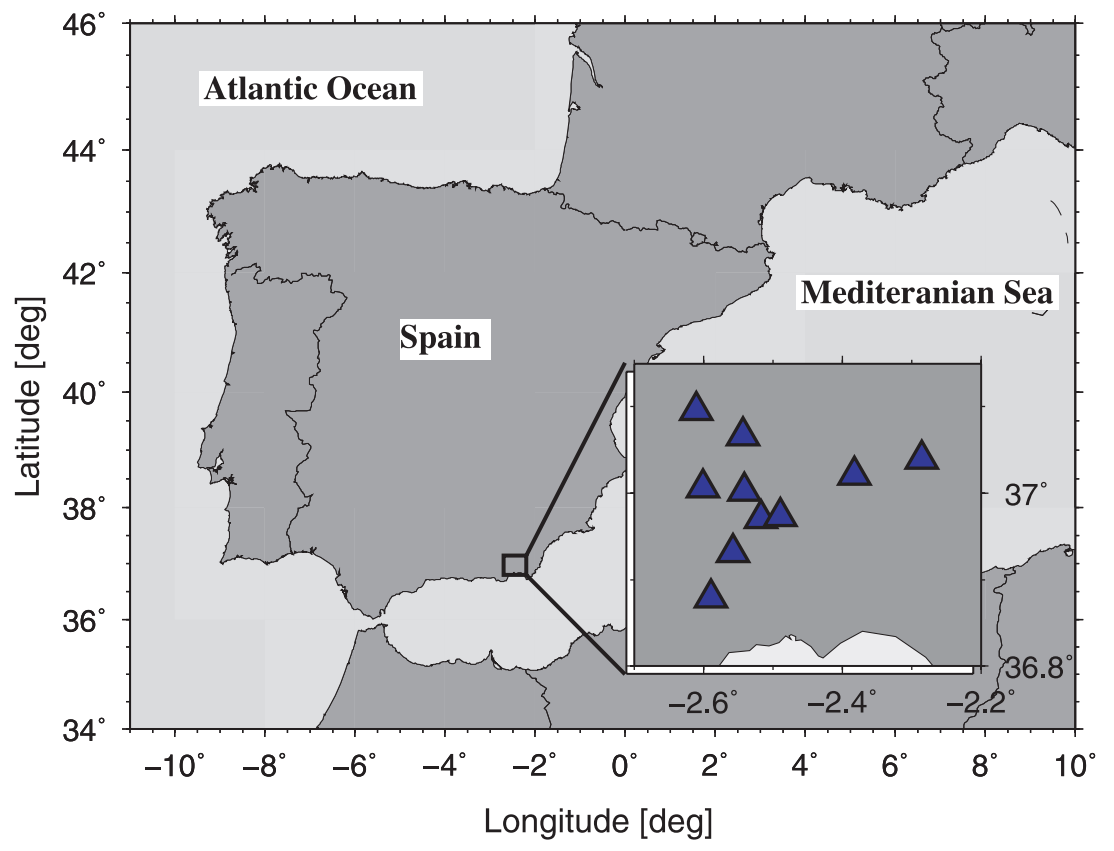

Figure 7. The temporary seismic array was deployed in the province of Almeria (South Spain) and consisted of 10 three-component broad-band sensors.

may explain waveform variations of cross-correlograms for neighbouring interstation distances.

The PCC section looks cleaner than the CCGN result which contains more energy at higher frequencies. The daily crosscorrelograms have also been stacked using the tf-PWS and the results for CCGN and PCC are shown in Figs 8(c) and (d). Power $v=2$ (eq. 7) and factor $k=2$ (eq. 5) were employed to obtain these results. The results were further cleaned from the noise with respect to the linear stack, as can be seen from a comparison of these figures. The improvement over the linearly stacked PCC traces is not as strong as over the CCGN traces since the PCCs (Fig. 8b) are already less contaminated by noise than the CCGNs (Fig. 8a).

The normalized amplitude spectra for two station pairs and the corresponding cross-correlograms are compared in Fig. 9. The 

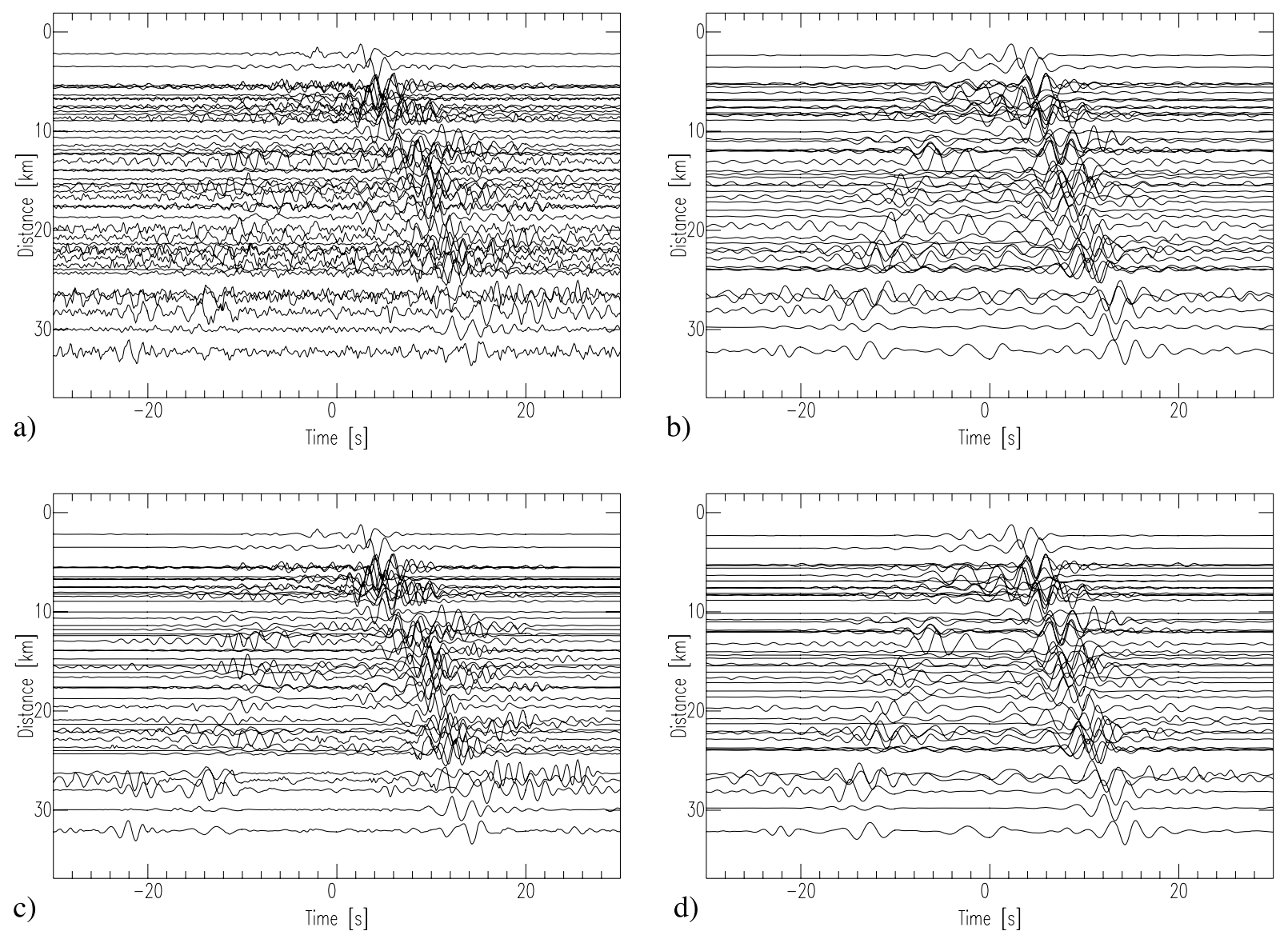

Figure 8. Ambient noise cross-correlation results for data recorded at the stations shown in Fig. 7. The daily cross-correlograms were computed and stacked using (a) CCGN and linear stack, (b) PCC and linear stack, (c) CCGN and tf-PWS and (d) PCC and tf-PWS. tf-PWS power $v=2$ were used.

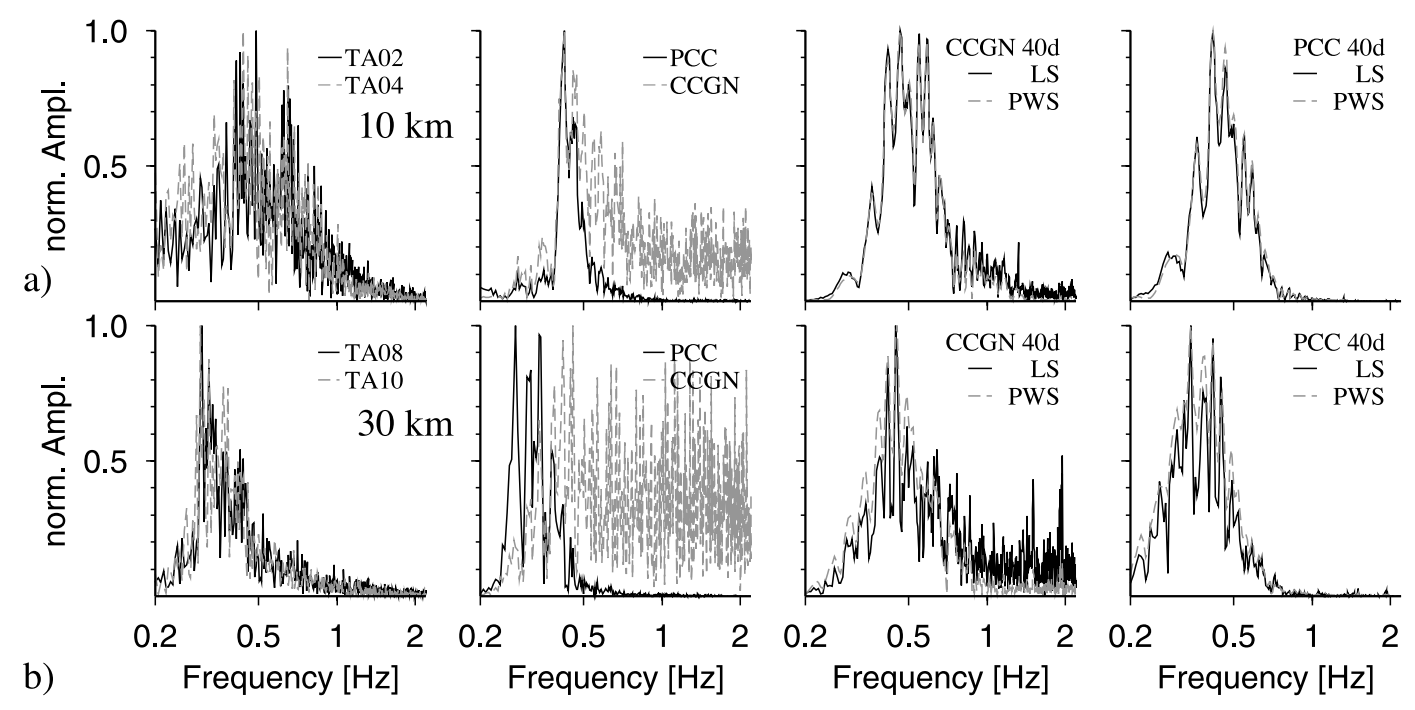

Figure 9. Noise and cross-correlation amplitude spectra for the two station pairs TA02-TA04 (a) and TA08-TA10 (b) with interstation distances $10 \mathrm{~km}$ and $30 \mathrm{~km}$ (Fig. 8). From left to right: The first panel shows the amplitude spectra of $200 \mathrm{~s}$ noise. The second panel contains the spectra for cross-correlations (PCC and CCGN) of $1 \mathrm{~d}$ of noise. See text for processing details. The third and fourth panel show the amplitude spectra after stacking 40 cross-correlations using CCGN and PCC, respectively. LS and PWS are abbreviations of linear stack and tf-PWS $(v=2)$. Lag times of all cross-correlograms range from $-100 \mathrm{~s}$ to $100 \mathrm{~s}$.

interstation distances are $10 \mathrm{~km}$ (Fig. 9a) and $30 \mathrm{~km}$ (Fig. 9b). TA02, TA04, TA08 and TA10 are station identifications. Figs 9(a) and (b) use data from different days. The first panel contains the amplitude noise spectra determined by a Fast Fourier Transform of the vertical component noise records. It is roughly similar at both stations. The second panel shows the PCC (solid line) and CCGN (grey line) amplitude spectra obtained for $1 \mathrm{~d}$ of noise with correlation lag time range $-100 \mathrm{~s} \leq t \leq 100 \mathrm{~s}$. The high-frequency 


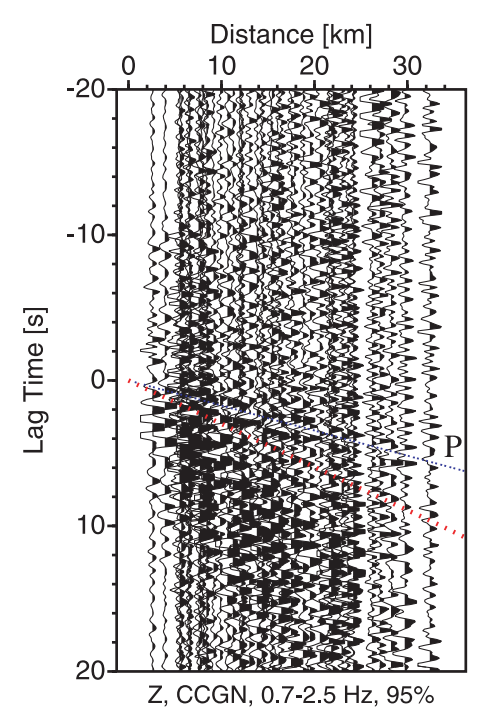

a)

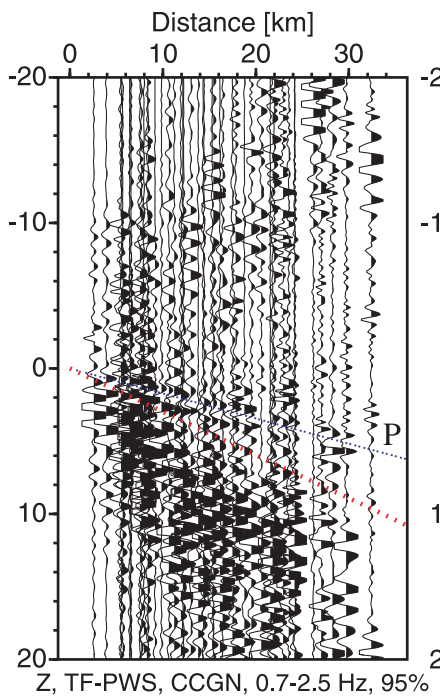

b)

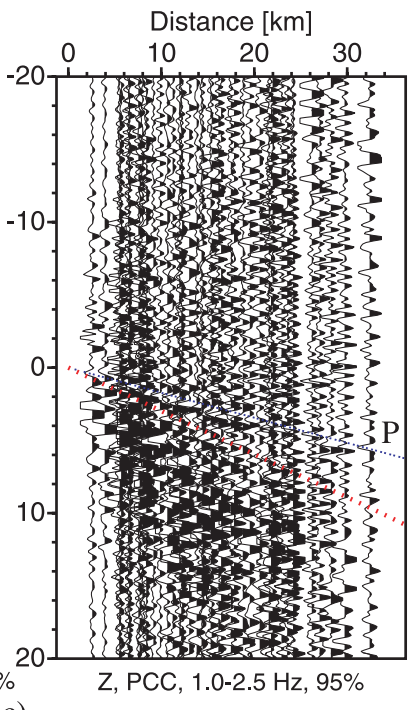

c)

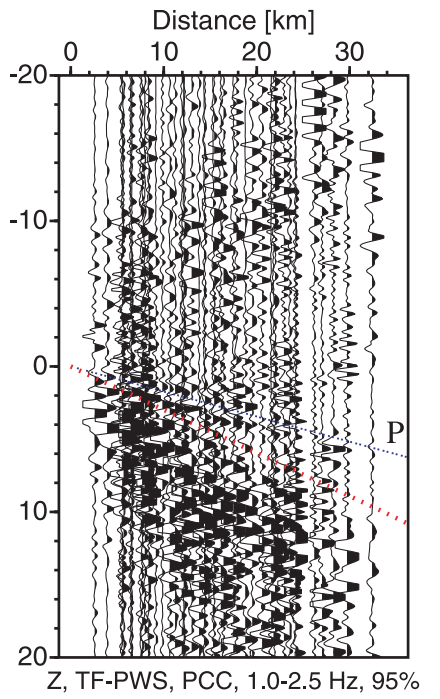

d)
Z, TF-PWS, PCC, 1.0-2.5 Hz, 95\%

Figure 10. Vertical component ambient noise cross-correlograms for the stations shown in Fig. 7. Record sections were obtained using (a) CCGN and linear stack, (b) CCGN and tf-PWS, (c) PCC and linear stack and (d) PCC and tf-PWS, respectively. tf-PWS power $v=2$ were used. The amplitudes are clipped at 95 per cent of the maximum amplitude on each trace. The theoretical $P$ - and $S$-phase traveltimes are shown as blue and red curves, respectively. $P$ phases are visible on all record sections.

signals in the CCGN spectra are due to the whitening of the data preprocessing. The last two panels contain the amplitude spectra of the linear stack (LS) and tf-PWS (PWS) of 40 CCGN and PCC traces, respectively. The LS and tf-PWS are shown with the solid and grey line. A comparison of these figures shows that the LS of the CCGN traces has higher frequencies than the LS of the PCC traces. We attribute this to the pre-processing of the CCGN data It is further seen that tf-PWS attenuates the higher frequencies of the CCGN time-series with respect to the LS. This high-frequent incoherent noise cleaning is not visible for PCC where the amplitude spectra remain similar. This is consistent with the little improvement archived with tf-PWS on the PCC traces as seen from Figs 8(b) and (d).

We observe in our ambient noise cross-correlograms also $P$ phases which are shown in Fig. 10. The plotting style was changed for this figure to increase the visibility of these less commonly observed body waves in regional scale settings with stations at the Earth's surface. In Fig. 10 each trace was clipped at 95 per cent of its maximum amplitude. The cross-correlograms were processed using CCGN and linear stack (10a), CCGN and tf-PWS (10b), PCC and linear stack (10c), PCC and tf-PWS (10d). The blue and red dashed lines mark the theoretical $P$ - and $S$-phase arrivals for a source at the Earth surface using the spherically symmetric seismic velocity model AK135 (Kennett et al. 1995). The $P$ phases line up with their theoretical traveltime curve. In other words the interstation distances correspond with the epicentral distances for $P$ waves generated at one of the stations used in the correlation. $S$ phases are not visible and their theoretical traveltimes are plotted for orientative purposes. The Rayleigh waves are visible after the $S$ arrival times.

The $P$ phases are recognized in all record sections from Fig. 10. The identification of the $P$ phases on the linearly stacked traces (Fig. 10a) alone is difficult. However, the incoherent noise cleaning using tf-PWS (Figs 10b and d) improves their visibility and increases their significance since tf-PWS attenuates signals if they do not appear with a certain regularity and coherence on the individual cross-correlograms.

We see also from Fig. 10 that $P$ and Rayleigh waves are mainly observed on the causal part of the cross-correlograms. Most of the noise wavefields are generated in the Mediterranean Sea which explains the observed dominant wave directions. A consistent observation of these signals can permit to do ambient noise body wave tomography, with the advantage that source positions and absolute traveltimes are well determined by the station position and the cross-correlation lag time.

\subsection{Real data: global-scale cross-correlations}

We use now globally distributed stations from the GEOSCOPE network to show the performance on long range noise crosscorrelations. For a detailed study of the noise characteristics of the global network see Stutzmann et al. (2009). Here, the continuous vertical component records were cut into $24 \mathrm{hr}$ long time-series and bandpass filtered with corner frequencies $0.003 \mathrm{~Hz}$ and $0.04 \mathrm{~Hz}$.

Then, daily cross-correlograms were computed between data from different station pairs using CCGN and PCC. The data used in the CCGN correlations were pre-processed by performing the 1bit normalization and spectral whitening while the PCC data were employed without any further pre-processing. The CCGN data was bandpass filtered after the spectral whitening.

The cross-correlograms for $1 \mathrm{yr}$ were stacked to build the record sections from Fig. 11 where distance is the interstation distance for each station pair. The station pairs used for this figure are written to the right-hand side of Figs 11(b) and (d). In Figs 11(a) and (b), we show the linearly stacked cross-correlations using CCGN (a) and PCC (b) while the corresponding tf-PWS are shown in Figs 11(c) (CCGN) and $d$ (PCC). The tf-PWS were computed with power $v=2$.

All record sections show the minor-arc Rayleigh waves which are labelled as R1 in Fig. 11(b). The corresponding major-arc waves R2 are only visible in the PCC sections of Figs 11(b) and (d). R2 Rayleigh waves travel between both stations using the larger great circle arc which complements the closed 360 degree circle.

It can be seen from the comparison of the linearly stacked traces with the corresponding tf-PWS cross-correlograms that the tf-PWS sections contain less noise. tf-PWS further improves the visibility of 

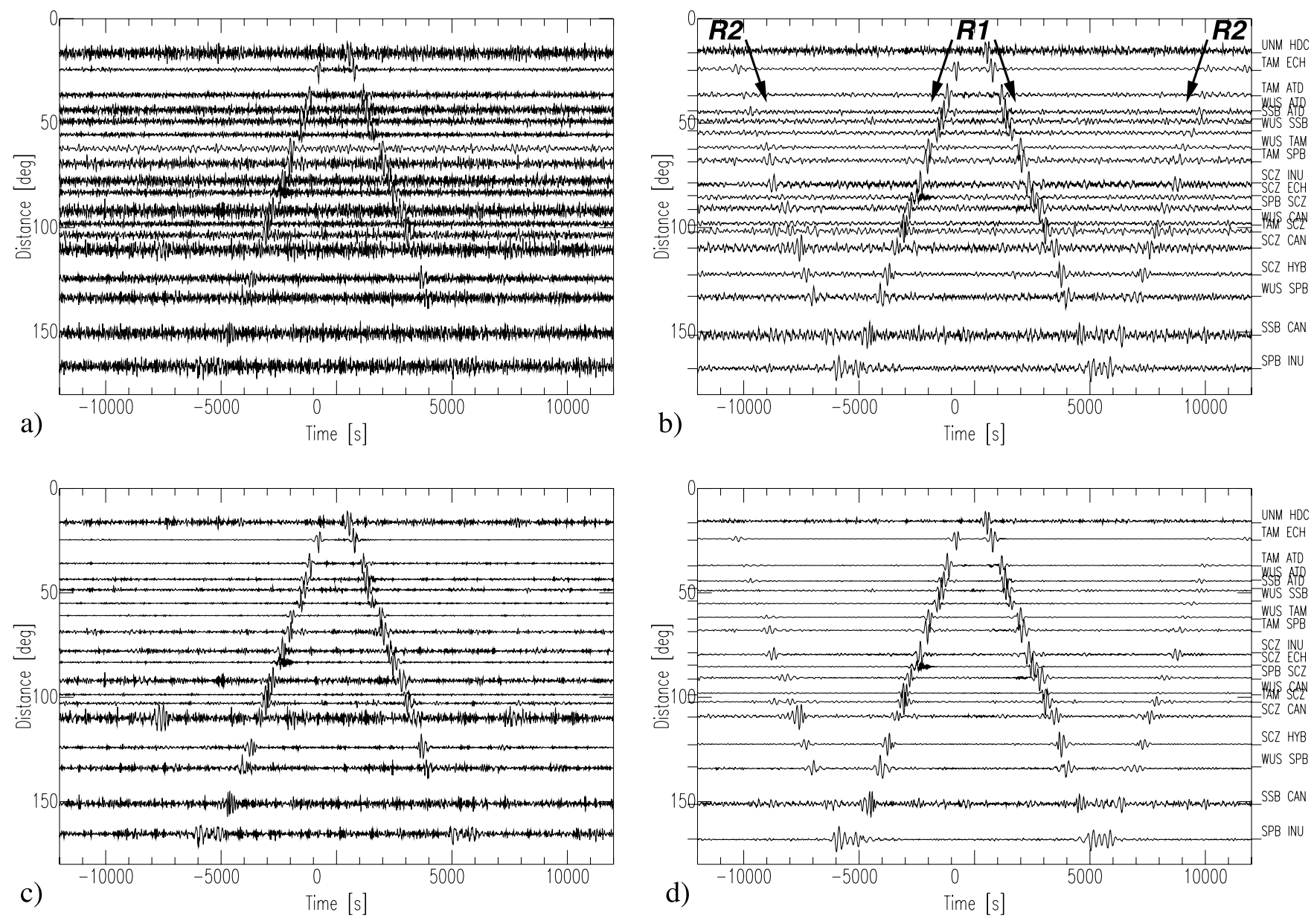

Figure 11. The daily cross-correlograms were computed and stacked for 1 yr of data using (a) CCGN and linear stack, (b) PCC and linear stack, (c) CCGN and tf-PWS and (d) PCC and tf-PWS. tf-PWS power $v=2$ were used. R1 and R2 mark the minor-arc and major-arc Rayleigh waves. Station pairs are written to the right of (b) and (d).

the signals. There are less high-frequency noise visible on the PCC traces than on the CCGN traces. This is due to the more sensitive phase coherence measure of PCC which finds less waveform similarities at the higher frequencies than CCGN. A $0.003-0.006 \mathrm{~Hz}$ bandpass filter reveals that R2 signals are also visible on the CCGN sections at lower frequencies as shown in Fig. 12. Nevertheless, a comparison between the CCGN and PCC shows that the bandpassed CCGN section does contain less R2 signals than the PCC section. The ambient noise at the frequencies used in this example are due to infragravity ocean waves (Nishida et al. 2009).

\section{DISCUSSION AND CONCLUSION}

We show that our processing tools improve the signal extraction from ambient noise data. The PCC is based on the phase coherence which we obtain from the phase of the analytic signals. Signals are therefore detected by their phase coherence while with the classical cross-correlations (CCGN) signals are identified by the largest sum of amplitude products, thus energy. This is the main difference between PCC and CCGN and both methods can therefore be considered as independent functionals. Based on their different philosophy the correlation results may differ due to signal and noise properties. If the signals should be detected by their waveform coherence then PCC is the better approach since it is the more sensitive measure as shown in Fig. 1 [more examples in (Schimmel 1999)]. Conversely, CCGN is amplitude biased and may not discriminate between closely similar waveforms.

Another important aspect of our analysis is the amplitude bias of the classical approach. In contrast to CCGN, PCC is not amplitude biased and therefore no special pre-processing is required to remove large amplitude events. The main advantage is not in omitting preprocessing steps, but is using as little as possible processed/altered waveforms for signal identification. For best results with CCGN the spectral whitening is often performed in a selected frequency band (e.g. Roux et al. 2005b) where coherent seismic noise is expected to be present. Our data (with or without pre-processing) were bandpass filtered into the frequency band where we expected to extract signals. We performed no quantitative analysis to select the frequency range. It is shown that under these conditions PCC provides the better results, thus being less sensitive to the frequency range than CCGN. This is seen in both examples, at local and global scale (Figs 8-12). The global scale example (Figs 11 and 12) shows that filtering the CCGNs to a low-frequency band increases the visibility of the R1 and $\mathrm{R} 2$ phases in the CCGN section. PCC still provides the better result due to the implicit data-adaptive coherence filtering. R1 and R2 phases were also shown by Nishida et al. (2009) who used stacks over $15 \mathrm{yr}$ of data and who used their dispersion curves for global tomography. Our figures are based on $1 \mathrm{yr}$ of data which is more than sufficient for PCC to extract clear R1 and R2 arrivals. 

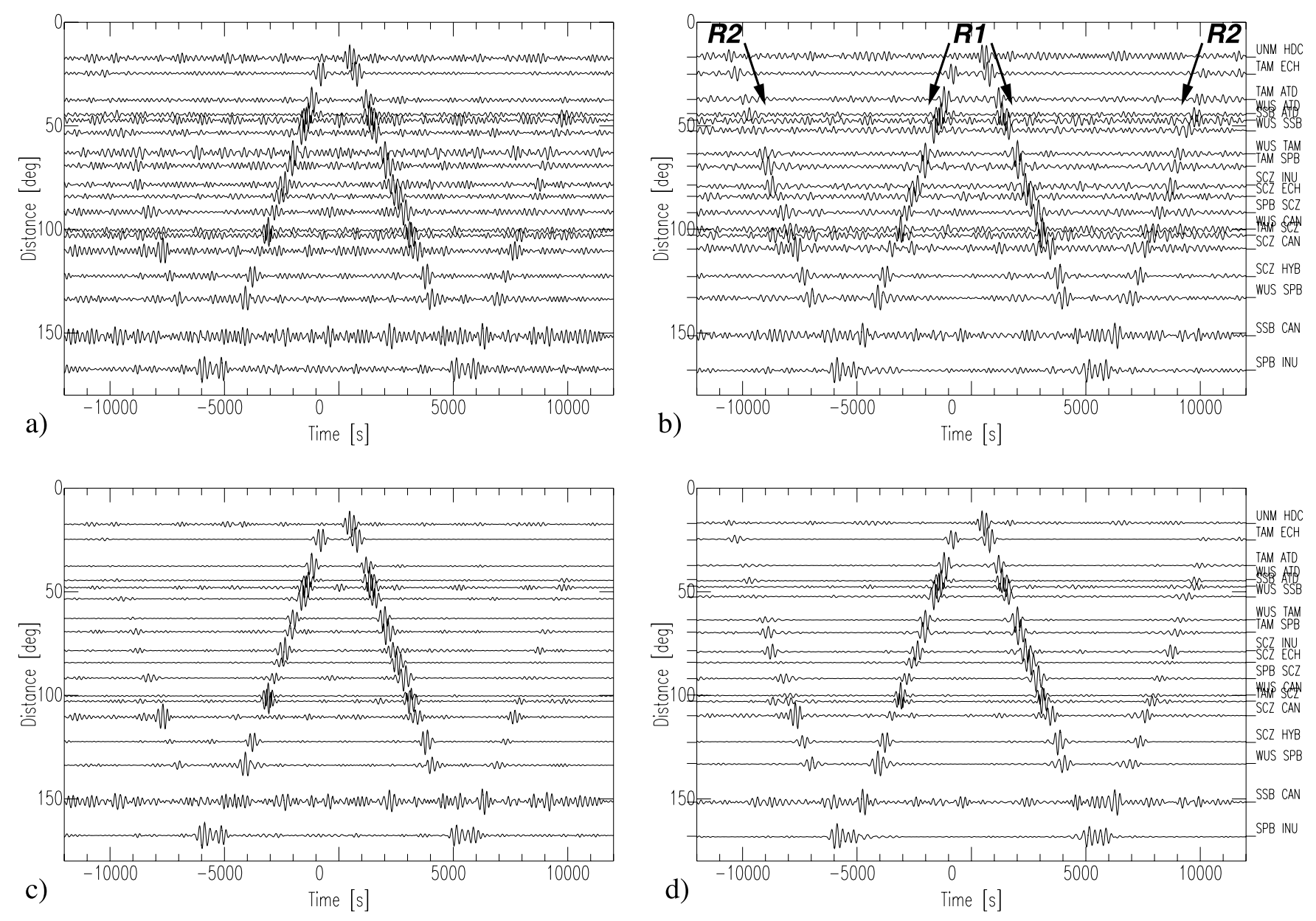

Figure 12. The cross-correlograms from Fig. 12 have been bandpass filtered between $0.003 \mathrm{~Hz}$ and $0.006 \mathrm{~Hz}$. R2 becomes now visible with CCGN, but still most R2 signals are detected with PCC.

The high waveform sensitivity of PCC can also turn into disadvantage. For instance, if the signal waveforms have high amplitudes and/or are very corrupted by noise then CCGN is expected to be the better approach. With PCC one can not expect to detect signals if they are not more phase-coherent than the noise. None of the correlations is understood to be the better approach. Their performance is related to their different strategies and depends on the signal and noise characteristics.

Another important processing step in the analysis of ambient noise data is the stacking of cross-correlograms. This way, the empirical Green's functions are emerging from the data over a larger time window which improves their SNR and the azimuthal coverage. A more efficient signal extraction may enable the use of shorter time windows which would increase the time resolution of monitoring studies. The incoherent noise attenuation during the stacking becomes therefore an attractive property. We use the tf-PWS (Schimmel \& Gallart 2007) which is a time-frequency domain extension of PWS (Schimmel \& Paulssen 1997). tf-PWS has also been used successfully by Baig et al. (2009) to show their benefits in the noise monitoring and signal extraction based on a non-redundant $S$-transform for the time-frequency representation. More generally, tf-PWS can be determined with any time-frequency representations which provides an analytic signal representation of the data in the time-frequency domain. Baig et al. (2009) show a significant improvement by identifying more Love and Rayleigh waves in their data. It is seen from our examples that the relative benefits of tf-PWS are larger when applied to CCGNs than to PCCs. This is because PCCs are already intrinsically cleaned from incoherent noise due to their larger waveform sensitivity. Nevertheless, tf-PWS can further remove incoherent noise or isolated signals on individual crosscorrelograms.

Our 35-km aperture array in South Spain permits the observation of $P$ waves in the cross-correlations as shown in Fig. 10. It is still rare to observe $P$ or $S$ phases from cross-correlations of ambient noise recorded at surface stations and separated by at least a couple of kilometres. They are more observed at higher frequencies and smaller scale studies such as performed in seismic exploration. Nevertheless, $P$ waves were also clearly observed by Roux et al. (2005b) and Nishida et al. (2008) at local and regional scale. Roux et al. (2005b) used stations located in an $11 \mathrm{~km}$ square and saw the $P$ waves up to $1.3 \mathrm{~Hz}$. Nishida et al. (2008) observes body waves up to distances of $400 \mathrm{~km}$ on cross-correlations of Hi-net tiltmeter recordings in Japan which were bandpass filtered from 0.02 to $0.5 \mathrm{~Hz}$. In our data we observe the $P$ waves at frequencies between 0.5 and $2.5 \mathrm{~Hz}$.

\section{ACKNOWLEDGMENTS}

GEOSCOPE is acknowledged for making its data available. This research has been supported by consolider-Ingenio 2010 program Nr. CSD2006-00041 (Topo-Iberia), PII200830I202 and the PN Nr. CGL2006-01171 (S-Iberia). MS acknowledges the IPGP-team for 
its invitations to several research stays. C. Corela is acknowledged to pointing to the writing mistake in eq. (13) of Schimmel \& Gallart (2007). The Editor A. Curtis and two anonymous reviewers provided constructive reviews which improved our manuscript. This is IPGP contribution number 3100 .

\section{REFERENCES}

Baig, A.M., Campillo, M. \& Brenguier, F., 2009. Denoising seismic noise cross correlations, J. geophys. Res., 114, B08310, doi:10.1029/ 2008JB006085.

Bakulin, A. \& Calvert, R., 2006. The Virtual Source method: theory and case study, Geophysics, 71, SI139-SI150.

Bensen, G.D., Ritzwoller, M.H., Barmin, M.P., Levshin, A.L., Lin, F., Moschetti, M.P., Shapiro, N.M. \& Yang, Y., 2007. Processing seismic ambient noise data to obtain reliable broad-band surface wave dispersion measurements, Geophys. J. Int., 169, 1239-1260, doi:10.1111/j.1365246X.2007.03374.x.

Brenguier, F., Shapiro, N.M., Campillo, M., Ferrazzini, V., Duputel, Z., Coutant, O. \& Nercessian, A., 2008. Towards forecasting volcanic eruptions using seismic noise, Nature Geosci., 1, doi:10.1038/ngeo104, 126130.

Campillo, M. \& Paul, A., 2003. Long-range correlations in the diffuse seismic coda, Science, 299, 547-549, doi:10.1126/science.1078551.

Chavez-Garcia, F.J. \& Rodriguez, M., 2007. The correlation of microtremors: empirical limits and relations between results in frequency and time domains, Geophys. J. Int., 171, 657-664, doi:10.1111/j.1365246X.2007.03529.x.

Derode, A., Larose, E., Tanter, M., de Rosny, J., Tourin, A., Campillo, M. \& Fink, M., 2003. Recovering the Green's function from field-field correlations in an open scattering medium, J. acoust. Soc. Am., 113, 2973-2976.

Duvall, T.L., Jefferies, S.M., Harvey, J.W. \& Pomerantz, M.A., 1993. Time distance helioseismology, Nature, 362, 430-432.

Holcomb, L.G., 1998. Spectral structure in the Earth's microseismic background between 20 and 40 seconds, Bull. seism. Soc. Am., 88(3), 744-757.

Kennett, B.L.N., Engdahl, E.R. \& Buland, R., 1995. Constraints on seismic velocities in the Earth from travel times, Geophys. J. Int., 122, 108-124.

Lobkis, O.I. \& Weaver, R.L., 2001. On the emergence of the Green's function in the correlations of a diffuse field, J. acoust. Soc. Am., 110, 3011-3017.

Nishida, K., Kawakatsu, H. \& Obara, K., 2008. Three-dimensional crustal S wave velocity structure in Japan using microseismic data recorded by Hinet tiltmeters, J. geophys. Res., 113, B10302, doi:10.1029/2007JB005395.

Nishida, K., Montagner, J.P. \& Kawakatsu, H., 2009. Global surface wave tomography using seismic hum, Science, 326, 5949, 112, doi:10.1126/science1176389.

Rickett, J. \& Claerbout, J., 1999. Acoustic daylight imaging via spectral factorization: helioseismology and reservoir monitoring. Leading Edge, 18, 957-960.

Rickett, J. \& Claerbout, J., 2000. Calculation of the acoustic solar impulse response by multi-dimensional spectral factorization. Solar Phys., 92(1/2), 203-210.

Roux, P., Sabra, K.G., Kuperman, W.A. \& Roux, A., 2005a. Ambient noise cross correlation in free space: theoretical approach, J. acoust. Soc. Am., 117, 97-84.

Roux, P., Sabra, K.G., Gerstoft, P. \& Kuperman, W.A., 2005b. P-waves from cross-correlation of seismic ambient noise, Geophys. Res. Lett., 32, L19303, doi:10.1029/2005GL023803.
Sabra, K.G., Gerstoft, P., Roux, P., Kuperman, W.A. \& Fehler, M., 2005. Surface wave tomography from seismic ambient noise in Southern California, Geophys. Res. Lett., 32, L14311, doi:10.1029/2005GL023155.

Schimmel, M., 1999. Phase cross-correlations: design, comparisons and applications, Bull. seism. Soc. Am., 89, 1366-1378.

Schimmel, M. \& Gallart, J., 2005. The inverse S Transform in filters with time-frequency localization, IEEE Trans. Signal Process., 53(11), 4417-4422, doi:10.1109/TSP.2005.857065.

Schimmel, M. \& Gallart, J., 2007. Frequency-dependent phase coherence for noise suppression in seismic array data, J. geophys. Res., 112, B04303, doi:10.1029/2006JB004680.

Schimmel, M. \& Paulssen, H., 1997. Noise reduction and detection of weak, coherent signals through phase weighted stacks, Geophys. J. Int., 130, 497-505, doi:10.1111/j.1365-246X.1997.tb05664.x.

Schuster, G.T., Yu, J., Sheng, J. \& Rickett, J., 2004. Interferometric/daylight seismic imaging, Geophys. J. Int., 157, 838-852.

Sens-Schönfelder, C. \& Wegler, U., 2006. Passive image interferometry and seasonal variations of seismic velocities at Merapi Volcano, Indonesia, Geophys. Res. Lett., 33, L21302, doi:10.1029/2006GL027797.

Shapiro, N.M. \& Campillo, M., 2004. Emergence of broadband Rayleigh waves from correlations of the ambient noise, Geophys. Res. Lett., 31, L07614, doi:10.1029/2004GL019491.

Shapiro, N.M., Campillo, M., Stehly, L. \& Ritzwoller, M.H., 2005. High resolution surface wave tomography from ambient seismic noise, Science, 307, 1615-1618.

Shapiro, N.M., Ritzwoller, M.H. \& Bensen, G.D., 2006. Source location of the $26 \mathrm{sec}$ microseism from cross-correlations of ambient seismic noise, Geophys. Res. Lett., 33, L18310, doi:10.1029/2006GL027010.

Simon, C., Ventosa, S., Schimmel, M., Heldering, A., Dañobeitia, J.J., Gallart, J. \& Manuel, A., 2007. The S-transform and its inverses: side effects of discretising and filtering, IEEE Transactions on Signal Processing, 55, 4928-4937, doi:10.1109/TSP.2007.897893.

Snieder, R., 2004. Extracting the Green's function from the correlation of coda waves: a derivation based on stationary phase, Phys. Rev. E, 69(4), 046610, doi:10.1103/PhysRevE.69.046610.

Snieder, R., Wapenaar, K. \& Wegler, U., 2007. Unified Green's function retrieval by cross-correlation: connection with energy principles, Phys. Rev. E, 75, 036103, doi:10.1103/PhysRevE.75.036103.

Stockwell, R.G., Mansinha, L. \& Lowe, R.P., 1996. Localization of the complex spectrum: the S transform, IEEE Trans. Signal Process., 44(4), 998-1001.

Stutzmann, E., Schimmel, M., Patau, G. \& Maggi, A., 2009. Global climate imprint on seismic noise, Geochem. Geophys. Geosyst., 10, Q11004, doi:10.1029/2009GC002619.

Ventosa, S., Simon, C., Schimmel, M., Dañobeitia, J.J. \& Manuel, A., 2008. S-transform from a wavelets point of view, IEEE Trans. Signal Process., 56, 2771-2780.

Wapenaar, K., 2004. Retrieving the elastodynamic Green's function of an arbitrary inhomogeneous medium by cross correlation, Phys. Rev., Lett., 93, 254301, doi:10.1103/PhysRevLett.93.254301.

Wapenaar, K., Slob, E. \& Snieder, R., 2006. Unified Green's function retrieval by cross-correlation, Phys. Rev. Lett., 97, 234301, doi:10.1103/PhysRevLett.97.234301.

Weaver, R.L. \& Lobkis, O.I., 2001. Ultrasonics without a source: thermal fluctuation correlations at MHz frequencies, Phys. Rev. Lett., 87, 134301, doi:10.1103/PhysRevLett.87.134301.

Wegler, U. \& Sens-Schönfelder, C., 2007. Fault zone monitoring with passive image interferometry, Geophys. J. Int., 168, 1029-1033. 